This is explained in the following manner: If we add to the precipitate fresh blood solution, this dissolves the precipitate partially, and with a sufficient quantity, totally. This indicates that before the nearly insoluble precipitate is formed there exists in the solution another saltlike product containing less metal ions than the precipitate. If now this first product be a very strong combination, we will have to add quantities of salt proportional to the concentration of the blood solution (always used in the quantity of 5 (c) before the nearly insoluble precipitate is formed.

In other words, the necessary quantity of agglutinating salt will be porportional to the concentration. Now the quantity of salt increases more slowly than the concentration, which indicates that there exists a chemical equilibrium between the two salt-like products, deriver from the blood solution and the ions of the precipitating salt. This behavior cannot be easily explained from the point of view of the colloidal school, just as with the analogrous behavior of the agglutinating quantities.

All the facts in this investigation give strong arguments in favor of the physical-chemical theory, according to which the observed phenomena are due to chemical processes similar to those encountered in general chemistry, and in which the chemical equilibria play an important rôle.

Noler, Physico-chmical Institete. stockhoim, June 12, 190.4.

\title{
ON THE NATURE OF PRECIPITATED COLLOIDS.
}

BY II. W. FOOTE.

Received July : 1908 .

In this article experimental evidence is given tending to show that precipitated ferric and aluminium hyclroxides nay be regarded as solutions of water in ferric or aluminium oxides or in one of their lower hydroxides or more generally that they are solutions of a liquid in a solid.

Much experimental work has already been done on substances of this class. Van Bemmelen in particular has shown by a large amount of very careful work that colloidal precipitates like ferric hydroxide, aluminium hydroxide and silicic acid are not compounds of definite composition. He calls them absorption compounds. He has recognized clearly that the moist precipitates contain both water mechanically mixed with the precipitate and absorbed water, though he gives no means of distinguishing between the two. ${ }^{1}$ He considers that the amount of absorbed water is dependent on the structure of the jelly and that this amount is changed by the method of formation, by time, heating, and by the presence of foreign stibstances. He makes the statement that hydrogels

'7. amorg. (hom., 13, 2+t; 8 , 2t 
cannot be considered as solid solutions ${ }^{1}$ but later makes the contradictory statement ${ }^{2}$ that they do behave as such. Hardy ${ }^{3}$ has suggested that the soluble gels, or heat-reversible gels, like agar are composed of a solution of agar in water and a solid solution of water in agar, and he has shown that the concentration of agar in water solution changes with the temperature. He states, however, that the "insoluble gels," like silicic acid or aluminium hydroxide, are not to be regarded as solid solutions.

The experiments described later show that a moist precipitated ferric or aluminium hydroxide free from other substances behaves in many ways like a mixture of two partially miscible liquids such as ether and water.

When ether and water are saturated with each other so that two liquid phases are present, the system in general has the following characteristics:

I. The composition of each liquid phase is constant at constant temperature and pressure, independent of the relative amounts of the two phases and independent of the method of preparation.

2. The composition of each phase changes with a change in temperature.

3. The vapor-pressures of both liquid phases are equal both as to total pressure and the pressure of each constituent.

If in the system of ether and water, the water is continually removed, the pressure will retain its original constant value as long as both liquid phases are present. When the water phase disappears, the pressure of the water vapor begins to fall, finally becoming zero when pure ether is left. The point where the vapor pressure begins to fall, indicates the disappearance of the water phase and the liquid left at this stage will be a saturated solution of water in ether. At constant temperature, the composition of the liquid at this point will be constant and it will change with a change in temperature.

Similarly with a moist precipitate of pure ferric hydroxide, if we assume a solid solution, there should be a saturated solution of water in ferric oxide and of ferric oxide in water. As the ferric oxide is practically insoluble in water, its concentration is zero, so the mixture would consist of the solid solution of water in ferric oxide mechanically mixed with water. The vapor pressure of this mixture would thus be equal to the vapor pressure of pure water and when water is removed, the vapor pressure should remain constant as long as free water is present. The vapor pressure should begin to fall at the point where the free water disappears and the composition of the residue at this point should give the composition of the saturated solution of water in ferric oxide. At

${ }^{1} Z$. anorg. Chem., 13, 234.

2 Ibid., 49, 137 .

${ }^{3} J$. Physiol, 24, 175 (1809). 
a constant temperature, different samples of ferric hydroxicle should show the same composition at the point where the vapor pressure begins to fall provided they have reached equilibrium with the water and this composition should change with a change in temperature.

To test these conclusions, two samples each of ferric hydroxide and aluminium hydroxide were prepared, using very different conditions for each of the two samples. In one case, 100 grams of crystallized aluminium chloride or ferric chloricle were dissolved in about six liters of cold water, using just enough hydrochloric acid to prevent a basic salt from forming. 'This cold dilute solution was precipitated with ammonia and thoroughly washed by decantation. In the other case, the samples were prepared by dissolving $I(x)$ grams of the same crystallized salts in one liter of hydrochloric acid, precipitating with ammonia and washing as before. The reaction between the ammonia and hydrochloric acid heated the solution nearly to the boiling point. (nne sample of each hydroxide, therefore, was precipitated from a cold dilute solution containing but little ammonium chloride, and the other was precipitated from a hot concentrated solution with a large excess of ammonium chloride. All the samples were washed very thoroughly, first by decantation and then on the filter until no test for chloride was ob tained when a little of the precipitate was dissolved and tested with silver nitrate. These precipitates after being prepared were allowed to stand in a moist condition several days at the temperature of the experiments. It was hoped that by this method the hydrates would reach a state of equilibrium with the water in contact with them. The composition of the moist precipitate was determined by ignition and a small weighed sample ( $\mathbf{I}-2$ grams) was placed in a weighed porcelain crucible and allowed to dry party in the air at the temperature of the experiment. A small pestle of glass or porcelain rod was weighed with the crucible and this was used to mix the precipitate frequently to prevent meven drying. At intervals, the crucible was weighed. then placed on a raised triangle in a large weighing bottle on the bottom of which was some pure water. The bottle was made tight with melted paraffin and sunk in a thermostat for from eighteen to forty-eight hours. The crucible was then remored and weighed quickly to determine the change in weight.

A preliminary experiment showed that a crucible containing pure water lost weight slightly when placed over water as described, although it might be expected that the weight would remain constant. 'The crucible contained 1.0560 grams of water and lost 0.0076 gram in about twenty-four hours. All the moist precipitates behaved similarly, always losing weight slightly over pure water, and this appears to be the normal behavior while mechanical water is present mixed with hydrates. After drying in the ait to a cortain point. howerer, the precipitates hegan 
to gain weight when placed over water. This was due to the fact that their vapor pressure had fallen below that of the water, showing that the water phase had just disappeared. The composition at the point where gain in weight first occurred gave approximately the composition of the saturated hydrate free from mechanical water. It may be of interest to state that the pcint where the material first began to absorb water could be very : closely determined by noting the mechanical condition of the precipitates, which appeared moist up to this point and dry beyond . The method outlined above. was used in every case but one-that of aluminium hydroxide at $25^{\circ}$. In this case, a considerable quantity of the moist hydroxide was slowly dried and samples removed from time to time and tested for loss or gain over water as before. This method was not as convenient and was harder to control.

The results obtained are given below. The composition at the point

FERric Hydroxide.

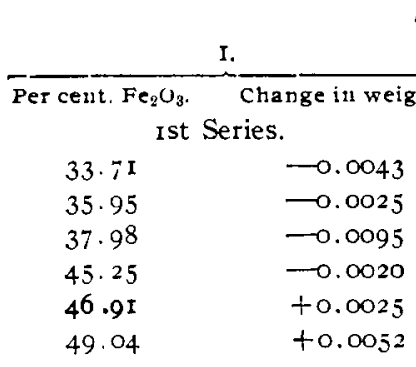

2nd Series.

$\begin{array}{ll}34.32 & -0.0040 \\ 42.80 & -0.0030 \\ 46.28 & -0.0015 \\ 48.53 & +0.0010 \\ 50.70 & +0.0050 \\ 54.74 & +0.0090 \\ & \text { T. }=45^{\circ} .\end{array}$

Ist Series.

$\begin{array}{ll}\mathbf{1} 4.99 & -0.0115 \\ 30.38 & -0.0230 \\ 52.68 & -0.0010 \\ 54.45 & +0.0020 \\ 55.62 & +0.0055\end{array}$

2nd Series.

$\begin{array}{ll}\mathbf{1 4} .90 & -0.0145 \\ 28.66 & -0.0200 \\ \mathbf{5 2 . 8 2} & -0.0015 \\ \mathbf{5 4 . 6 0} & +0.0035\end{array}$

$$
\text { T. }=25^{\circ} \text {. }
$$$$
\text { Per cent. } \mathrm{Fe}_{2} \mathrm{O}_{3} \text {. Change in weight. }
$$

Ist Series.

$\begin{array}{ll}23.21 & -0.0065 \\ 31.26 & -0.0125 \\ 37.13 & -0.0060 \\ 40.00 & -0.0045 \\ 44.94 & -0.0015 \\ 46.35 & +0.0010 \\ 50.18 & +0.0035\end{array}$

and Series.

$\begin{array}{ll}23.72 & -0.0040 \\ 32.97 & -0.0060 \\ 41.60 & -0.0030 \\ 46.09 & -0.0020 \\ 49.23 & +0.0025 \\ 56.43 & +0.0061\end{array}$

Ist Series.

$15 \cdot 74$

25.53

$4 \mathbf{I} \cdot 79$

53.81

$55 \cdot 77$

$57 \cdot 18$

and Series.

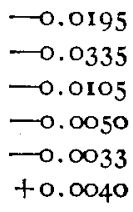

I6.3I -0.0145

$33.78 \longrightarrow 0.0195$

$53 \cdot 92$

55.22

$\mathbf{5 6 . 3 0}$

59.66
$-0.0050$

$\pm 0.0000$

$+0.0017$

$+0.0060$ 
where gain in weight first occurred is printed in heavy type. The results under I were obtained with precipitates formed from cold dilute solutions. Under II are the results from hot concentrated solutions. Duplicates, called first and second series, were carried out in each case. In calculating the composition, the weight of the oxide was determined by ignition at the end of a series of determinations. The weight of the precipitate was found by subtracting the weight of the empty crucible from the weight of the crucible with the precipitate afier it had been over water in the thermostat. The approximate composition of the material at any point could also be calculated as the composition of the original moist precipitate was known with some degree of accuracy from analysis of similar material.

Aluminium Hydroxide.

\begin{tabular}{|c|c|c|c|}
\hline & 1. & & I. \\
\hline Percent. $\mathrm{Al}_{2} \mathrm{O}_{3}$ & Change in weight. & Per cent. $\mathrm{Al}_{2} \mathrm{O}_{3}$. & Change in weigh \\
\hline \multicolumn{2}{|c|}{ Ist Series. } & \multicolumn{2}{|c|}{ Ist Series. } \\
\hline 43.13 & -0.0075 & 41.57 & -0.0010 \\
\hline 45.60 & -0.0025 & $4^{8} \cdot 47$ & $+0.008,3$ \\
\hline $49 \cdot 97$ & -0.0060 & & \\
\hline 51.42 & +0.0226 & & \\
\hline \multicolumn{2}{|c|}{ and Series. } & \multicolumn{2}{|c|}{ 2nd Series. } \\
\hline 32.47 & -0.0055 & $44 \cdot 74$ & -0.0009 \\
\hline 42.26 & -0.0098 & $50 \cdot 57$ & +0.0148 \\
\hline 46.18 & $\longrightarrow .0055$ & 52.70 & +0.0450 \\
\hline 48.25 & -0.0040 & & \\
\hline 50.91 & +0.0035 & & \\
\hline \multicolumn{4}{|c|}{$\mathrm{T} .=45^{\circ}$. } \\
\hline \multicolumn{2}{|c|}{ 1st Series. } & \multicolumn{2}{|c|}{ Ist Series. } \\
\hline 50.91 & -0.0080 & 51.39 & -0.0013 \\
\hline 52.70 & $\div 0.0080$ & 53.86 & $+0.004 \pi$ \\
\hline 53.01 & +0.0500 & & \\
\hline \multicolumn{2}{|c|}{ 2nd Series. } & \multicolumn{2}{|c|}{ 2nd Series. } \\
\hline 5 I. 56 & -0.0085 & 49.98 & 0.0053 \\
\hline $5^{2} .43$ & +0.0085 & 51.96 & +0.0005 \\
\hline \multirow[t]{3}{*}{$53 \cdot 39$} & +0.0095 & 53.27 & -0.0035 \\
\hline & & 53.77 & +0.0045 \\
\hline & & 55.93 & $+0.005 z$ \\
\hline
\end{tabular}

The method, it will be seen, can give only approximately the compo. sition of the residue when it first begins to gain water, but the duplicate results indicate that the results obtained are not largely in error and they appear to be accurate enough for the purpose. The fact that the losses in weight are irregular in the table, is chiefy because the length of time that the samples remained over water was not alike, the object being to determine whether there was a loss in weight and not how great the loss might be. 
The summary below gives the average results obtained when the residues first absorbed water, or in other words, when the residues have approximately the composition of the saturated solution.

FERRIC HYDRATE.

$\begin{array}{ccc}\text { Temperature. } & \text { Percent. } \mathrm{Fe}_{2} \mathrm{O}_{3} . & \text { II. } \\ 25^{\circ} & 47.72 . & \text { Per cent. } \mathrm{Fe}_{2} \mathrm{O}_{3} . \\ 45^{\circ} & 54.53 & 47.79 \\ & \text { ALUMINIUM HYDRATE. } & 56.74 \\ & \mathrm{I} . \mathrm{A} & \\ \text { Temperature. } & \text { Per cent. } \mathrm{Al}_{2} \mathrm{O}_{3} . & \text { Per cent. } \mathrm{Al}_{2} \mathrm{O}_{3} . \\ 25^{\circ} & 5 \mathrm{r} .17 & 49.52 \\ 45^{\circ} & 52.57 & 53.82\end{array}$

Different samples, prepared under widely different conditions, have nearly the same composition at the same temperature, indicating that the moist precipitates have approximately reached equilibrium with their surroundings. The composition changes with the temperature.

Certain objections may of course be made to the statement that ferric hydrate is to be regarded as a solution of water in ferric oxide. If the oxide is treated with water, the latter is not taken up to form the hydroxide. Again if part of the dissolved water is removed from the hydroxide by evaporation, this water is never completely taken up again. Van Bemmelen's results have shown this repeatedly and in my own experiments, I noticed that after a hydroxide was slightly dried and then exposed to water vapor, it never absorbed water enough to become saturated. While I have no experimental evidence to offer in explanation of this behavior, it is in general true that those reactions in which a solid directly takes part are often very slow, and the action may be so slow that it practically never takes place. It may also be true that the hydrates are not solutions of water in the oxide but a solution of water in some lower hydrate, a point which has been emphasized by Van Bemmelen in considering the hydrates as absorption compounds.

Another objection is in regard to the rate at which the moist precipitate dries in the air. It has been shown by Van Bemmelen that when a moist hydrate is allowed to dry spontaneously, there is no sharp change in the rate of evaporation. Such a change would in general be expected at the point where the free water has disappeared and dissolved water begins to go off. I believe the reason that no such change in rate has been observed is due to the conditions of the experiment. In order that the rate of evaporation from the moist precipitate shall be uniform, the material must not be stirred or disturbed. As a result, the part of the hydrate in contact with the air would lose water rapidly and some of the dissolved water would be removed while the parts out of contact 
with the air were still moist. A curve representing the loss by evaporation with the time in this case would not show a sharp change in direction.

Mr. M. S. Fine has carried out some experiments in this laboratory with zirconium hydroxide, using the same methods that were used with ferric and aluminium hydroxides. Iack of time has prevented him from completing the experiments, but his results as far as obtained agree with the previous ones. A solution containing very pure zirconium chloride with a slight excess of hydrochloric acid. was divided into two parts. I was diluted to five liters with water and precipitated with ammonia. II was diluted with one liter of hydrochloric acid and precipitated with concentrated ammonia, the reaction heating the solution nearly to boiling. The conditions are similar to those adopted in preparing the other hydroxides. Freliminary determinations had shown the approximate composition of the saturated zirconium hydroxide. His results were as follows:

\begin{tabular}{|c|c|c|c|}
\hline \multicolumn{4}{|c|}{$T=25^{\circ}}$. \\
\hline Per cent. $\mathrm{ZrO}_{2}$ & Change in weight. & Per cent. $\mathrm{ZrO}_{\mathrm{g}}$. & Change in weight. \\
\hline \multicolumn{2}{|c|}{ Ist Series. } & \multicolumn{2}{|c|}{ Ist Series. } \\
\hline 30.24 & -0.0022 & $32 \cdot 30$ & $\longrightarrow 0.0020$ \\
\hline $3 I .80$ & +0.0012 & 32.72 & +0.0008 \\
\hline \multicolumn{2}{|c|}{ 2nd Series. } & \multicolumn{2}{|c|}{ 2nd Series. } \\
\hline 32.26 & -0.0010 & 27.20 & $-0.002 \xi$ \\
\hline \multirow[t]{4}{*}{32.85} & +0.0007 & 30.80 & $\div 0.0010$ \\
\hline & & \multicolumn{2}{|c|}{ 3rd Series } \\
\hline & & $29 \cdot 40$ & \pm 0.0000 \\
\hline & & 32.54 & +0.0040 \\
\hline
\end{tabular}

The averages of his results for the composition of the saturated solution of water in zirconia are 32.33 and 32.02 per cent. of zirconia for $I$ and II respectively.

As before, the composition of the saturated solutions was nearly the same in both cases on standing in contact with water, although the original precipitates were prepared under very different conditions.

I believe the results given in this article are, with the exception of Hardy's results, the first quantitative evidence of any case of a solution of a liquid in a solid.

I propose to continue this work in the future.

ShRFField LABORATORY, NEW HAVEN, CONN,, June 18,1908 . 